\title{
Article \\ Noble Metallic Pyramidal Substrate for Surface-Enhanced Raman Scattering Detection of Plasmid DNA Based on Template Stripping Method
}

\author{
Wenjie Wu ${ }^{1}{ }^{1}$, Rui Li ${ }^{1, *}$, Maodu Chen ${ }^{1, *}$, Jiankang $\mathrm{Li}^{1}{ }^{1}$, Weishen Zhan ${ }^{1}$, Zhenguo Jing ${ }^{1}$ and Lu Pang ${ }^{2}$ \\ 1 School of Physics, Dalian University of Technology, Dalian 116000, China; wuwenjie@mail.dlut.edu.cn (W.W.); \\ xjljk@mail.dlut.edu.cn (J.L.); zhanwsh@dlut.edu.cn (W.Z.); jingzg@dlut.edu.cn (Z.J.) \\ 2 School of Materials Science and Engineering, Dalian University of Technology, Dalian 116000, China; \\ panglu@mail.dlut.edu.cn \\ * Correspondence: rli@dlut.edu.cn (R.L.); mdchen@dlut.edu.cn (M.C.)
}

Citation: Wu, W.; Li, R.; Chen, M.;

Li, J.; Zhan, W.; Jing, Z.; Pang, L.

Noble Metallic Pyramidal Substrate for Surface-Enhanced Raman

Scattering Detection of Plasmid DNA Based on Template Stripping Method. Micromachines 2021, 12, 923. https:// doi.org/10.3390/mi12080923

Academic Editor: Ruffino Francesco

Received: 14 July 2021

Accepted: 28 July 2021

Published: 2 August 2021

Publisher's Note: MDPI stays neutral with regard to jurisdictional claims in published maps and institutional affiliations.

Copyright: (C) 2021 by the authors Licensee MDPI, Basel, Switzerland. This article is an open access article distributed under the terms and conditions of the Creative Commons Attribution (CC BY) license (https:// creativecommons.org/licenses/by/ $4.0 /)$.

\begin{abstract}
In this paper, a new method for manufacturing flexible and repeatable sensors made of silicon solar cells is reported. The method involves depositing the noble metal film directly onto the $\mathrm{Si}$ template and stripping out the substrate with a pyramid morphology by using an adhesive polymer. In order to evaluate the enhancement ability of the substrate, Rhodamine 6G (R6G) were used as surface-enhanced Raman scattering (SERS) probe molecules, and the results showed a high sensitivity and stability. The limit of detection was down to $10^{-12} \mathrm{M}$ for R6G. The finite-difference time domain (FDTD) was used to reflect the distribution of the electromagnetic field, and the electric field was greatly enhanced on the surface of the inverted pyramidal substrate, especially in pits. The mechanism of Raman enhancement of two types of pyramidal SERS substrate, before and after stripping of the noble metal film, is discussed. By detecting low concentrations of plasmid DNA, the identification of seven characteristic peaks was successfully realized using a noble metallic pyramidal substrate.
\end{abstract}

Keywords: template stripping method; adhesive polymer; pyramid; surface-enhanced Raman spectroscopy (SERS); plasmid DNA

\section{Introduction}

Surface-enhanced Raman spectroscopy (SERS) is a potent noninvasive spectroscopy technology that can detect and characterize small organic molecules and large biomolecules at very low concentrations and at a single-molecule level. Molecular identification in a lowconcentration sample aqueous solution provides the potential for the unlabeled detection and identification of various analytes [1-4]. Since the discovery of SERS on the surface of noble metals, a new era of Raman spectroscopy has emerged. Metallic substrates with periodic subwavelength structures exhibit unique surface isobaric properties, providing an enhanced electromagnetic field and producing a strong optical response [5-7], resulting in SERS, which is of great scientific significance and considerable technical importance for the development of nanophotonic devices, data storage, and biosensors. As such, noble metallic nanoparticles already have widespread applications in creating local surface plasmon resonance (LSPR). By adjusting the geometry and optical properties of the nanoparticles, the Raman signal is greatly enhanced [8-10]. Hence, to control nanostructures and generate a larger local EM field, the design and manufacture of ordered and uniform nanostructured SERS substrates has become critical. In the past thirty years, solid support matrices with SERS effects, such as nanocolloids [11], rough metal surfaces [12], and periodic nanostructures [13-15], have been widely reported in the literature [16,17]. However, their limited stability and reproducibility often limit their practical applications $[18,19]$. Various techniques have been reported for the manufacture of uniform nano-SERS substrates with 
controllable periods, sizes, and geometries, such as focused ion beam lithography [20], electron beam lithography [21-24], and nanoindentation [25-27]. However, these techniques are expensive, time-consuming, and difficult to reproduce for large quantities [28,29].

In this study, high sensitivity SERS substrates were prepared using a template stripping method. We adopted anisotropic etching of crystalline silicon solar cell substrates as nanostructured surface templates with sharp edges, such as commercially available pyramid arrays [30], deposited a certain thickness of noble metal film in the silicon pyramid gap, and then applied a UV-curable polymer (UVcp) on the surface [31]. UVcp is a kind of transparent colloid that is composed of polyurethane acrylate. Under the irradiation of UV light, the UVcp on the surface of the noble metal film crosslinks to form a network structure to achieve stable binding. After curing, UVcp becomes a flexible polymer, and due to the poor adhesion between the noble metal and the silicon surface, the noble metal film deposited on the silicon template can be easily stripped by UVcp to form a densely arranged nanostructured noble metallic pyramidal substrate (PS) [32-35]. Due to the stability of silicon, the template is highly reproducible, reusable, and mass-producible $[36,37]$. The good flexibility of the UVcp used in the stripping of the noble metal film improves the load bearing capacity of the bonding surface [38], the damping characteristics are improved, and stress cracking will not occur at the typical molding stress level [39]. It can not only provide a large angle of curvature to adapt to various surfaces, but also enable the collection of Raman signals in situ because of its transparency [40]. The large Young's modulus allows the metallic features to avoid deformation during the stripping process and ensures structural integrity.

The structure before stripping of the metal film is called a positive pyramidal substrate (PPS), while the structure after stripping is called an inverted pyramidal substrate (IPS). The bottom gap of the pyramid array and the pyramidal side wall lead to a strong electromagnetic field enhancement, resulting in a rich and uniform SERS hot pots [41]. We carried out a numerical simulation and provide experimental evidence to affirm the excellent sensitivity, outstanding uniformity, and stability of the noble metallic PS, and also applied it to biological detection. As deoxyribonucleic acid (DNA) is an important molecule in biology that carries the genetic information for the growth, development, function, and reproduction of organisms [42], the detection of specific DNA sequences in humans, viruses, bacteria, and plants has becoming vital in various fields [43]; while also being helpful for the early diagnosis and prevention of diseases. In this paper, using $\mathrm{Ag} / \mathrm{IPS}$ as a SERS substrate, we achieved the low-concentration detection of plasmid DNA and the identification of seven characteristic peaks.

\section{Materials and Methods}

\subsection{Instrument}

SERS signals were gathered by a Raman spectrometer (Renishaw InVia, London, England) with an inverse microscope (Zeiss Axiover 25, Jena, Thuringia, Germany) and several filters (Renishaw InVia, London, England, $532 \mathrm{~nm}$, Cut off: $100 \mathrm{~cm}^{-1}, \mathrm{OD}=8$ ), and SERS spectra were acquired at room temperature. An x-y translational stage was used to adjust the samples with a micron-scale resolution (Renishaw RCH24, London, England). A 532nm laser (75mW, NOVAPRO, RGB Lasersystems, Kelheim, Bavaria, Germany) was employed for the incident light, which was focused onto the sample using a $50 \times$ objective (Nikon, MUL03501, Tokyo, Japan) lens. The accumulation time for each spectrum reported was $1 \mathrm{~s}$, and the resolution of all spectra was $1 \mathrm{~cm}^{-1}$.

Scanning electron microscopy (SEM) images were taken with an SEM system (SUPARR 55, Zeiss, Jena, Thuringia, Germany). A vacuum magnetron sputtering system (JCP-200, BTSC563, Beijing, China) was used to produce metal nanofilms. UVcp (AA3311, LOCTITE, Shanghai, China) was used for stripping the metallic nanostructures.

Rhodamine 6G (R6G) (R4127-100g, SIGMA, St. Louis, MO, USA) was selected as the probe molecule to test the SERS performance of the PS. The metallic PS were immersed in R6G solutions of different concentrations for $1 \mathrm{~h}$ and left to air dry at room temperature 
for more than $30 \mathrm{~min}$; the samples were used to measure the Raman spectrum. In order to illustrate the biological application of the enhancement substrate, plasmid DNA was chosen as the detected substance.

\subsection{Preparation of Plasmid DNA to Be Measured}

Plasmid DNA was transformed into DH5 $\alpha$ competent cells, the bacterial cultures were grown in 5mL LB (Luria-Bertani, Bioworld, Bloomington, IL, USA) broth for $16 \mathrm{~h}$ at $37{ }^{\circ} \mathrm{C}$, and finally plasmid DNA was extracted using miniprep (plasmid Miniprep Kit, Axygen, Union City, CA, USA), while the concentration of the plasmid was $120 \mathrm{ng} / \mu \mathrm{L}$.

\subsection{Fabrication of Noble Metallic PS}

To strip the metallic nanofilm structure onto a flexible substrate, UVcp was used, to obtain a high-fidelity and high-sensitivity sensor. A regular surface textured crystalline silicon solar cell substrate was chosen as the master mold, which placed random pyramids on the surface [44], as shown in Figure 1a; the substrate is about the size of a coin. A noble metal film with a thickness of $200 \mathrm{~nm}$ was deposited on the surface of the substrate, as shown in Figure 1b,c, then the UVcp was dropped onto the surface of the noble metal film. Before curing, UVcp as a solvent could flow and fill the PS at nano- and microscales, as shown in Figure 1e. The UVcp was cured with UV irradiation at $365 \mathrm{~nm}$ for $30 \mathrm{~s}$ at room temperature. Then the UVcp was stripped with tweezers and the noble metallic structure was successfully stripped onto the flexible UVcp substrate. Figure $1 d$,f show before and after the process of stripping, respectively. The reason for the curling of the nanofilm is its flexibility, due to the soft UVcp substrate. Au and Ag were selected as the noble metals for this work, which resulted in four different substrates: (b) Ag/PPS, (c) Au/PPS, (f) Ag/IPS, and Au/IPS. The process of the proposed method is simple and can realize rapid measurement and protect the substrate surface from oxidation.

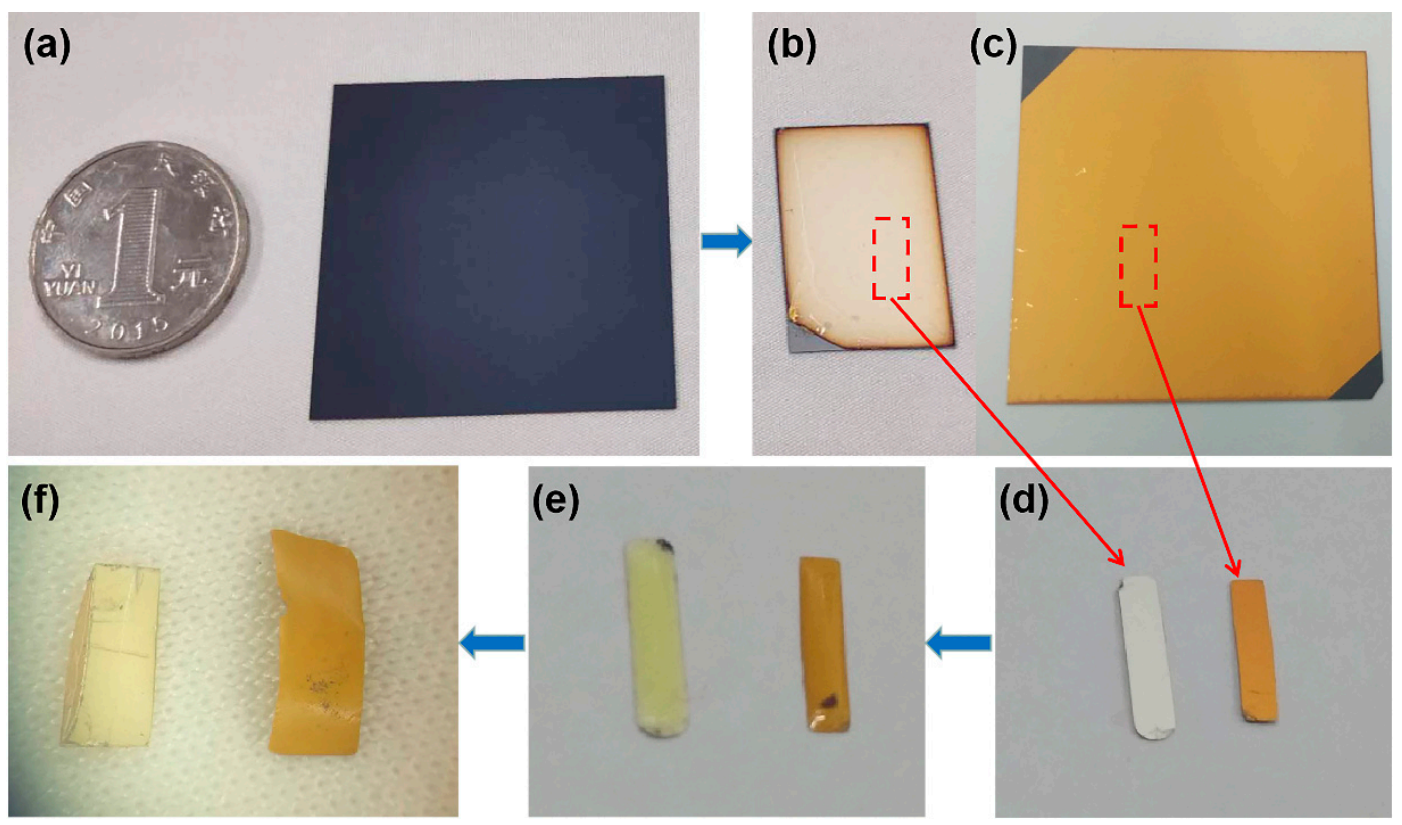

Figure 1. (a) Regular surface textured crystalline silicon solar cell substrate; (b) Au/PPS; (c) Ag/PPS; (d) Au/PPS; and Ag/PPS after cutting; (e) UVcp was applied to (d); (f) the Ag/IPS and Au/IPS.

\subsection{Scanning Electron Microscopy Imaging of Pyramidal Structures}

To study the morphological characteristics of the PPS and IPS, SEM was used to image the morphology of the nanostructure before and after stripping onto the UVcp substrate. Figure 2 shows top-view SEM micrographs of the four kinds of noble metallic pyramidal substrates at different magnifications. Figure $2 \mathrm{a}-\mathrm{d}$ shows the high uniformity and evenness 
of the pyramidal substrates at micron scale, which reflects the stability of the vacuum magnetron sputtering machine. Figure 2e-h shows top-view SEM micrographs of the two noble metal films after successful stripping. Au and Ag films underwent the same stripping process under the same environment. From the enlarged image, the surface of the stripped metal film is seen to be rough and uneven, and not as smooth as before. This is because there is a certain adhesion between the metal film and silicon during the stripping process, which means that the metal film cannot be completely stripped. In addition, the morphology of the IPS obtained by the two noble metals is also different, which is due to the different adhesions of the $\mathrm{Au}$ and $\mathrm{Ag}$ films to the silicon substrate.

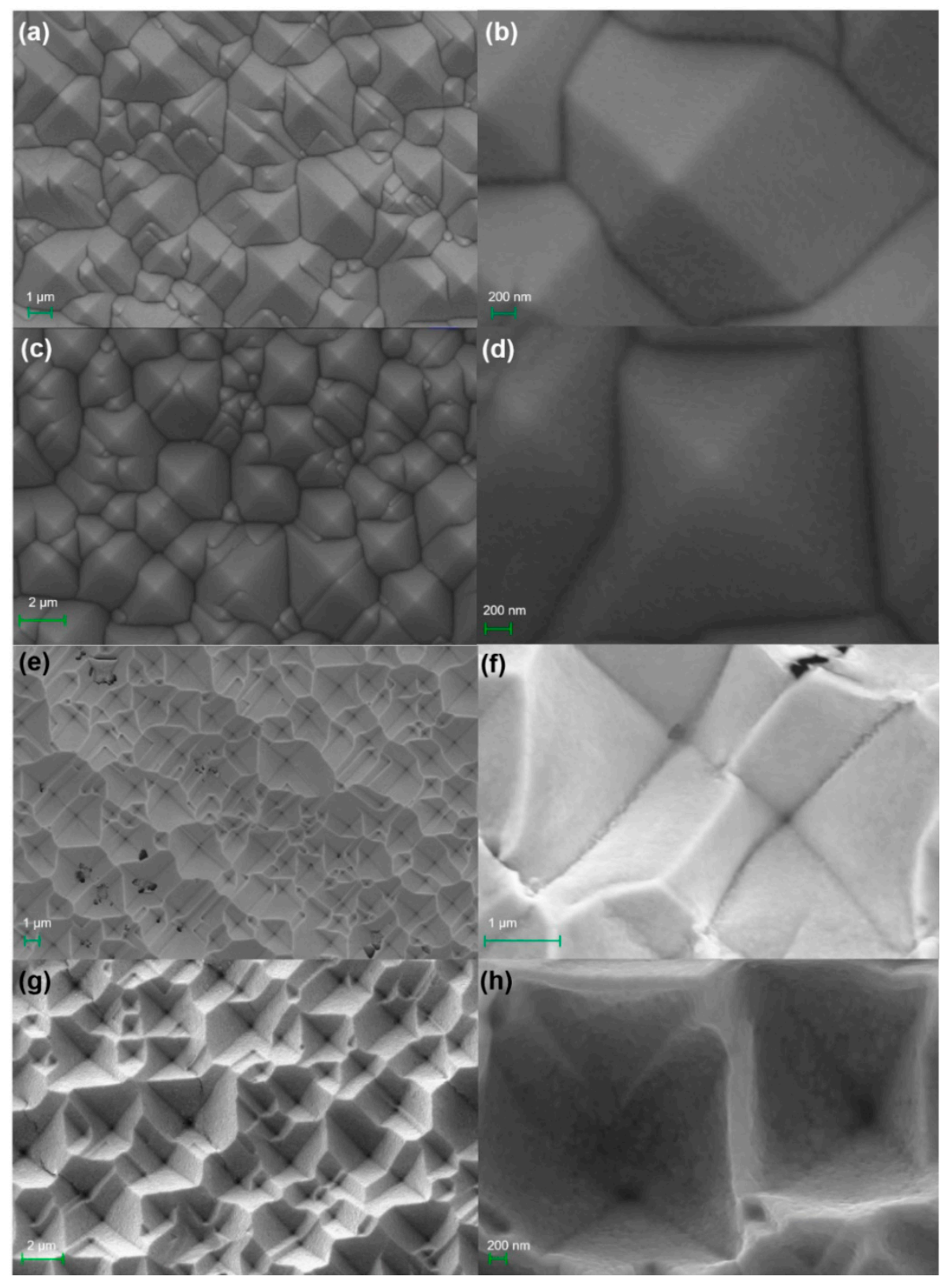

Figure 2. SEM images of four kinds of SERS substrate at different magnifications. (a) and (b) Au/PPS; (c) and (d) Ag/PPS; (e) and (f) Au/IPS; (g) and (h) Ag/IPS.

\section{Results and Discussion}

\subsection{Simulation Analysis of Electric Field Distribution Using PS}

To clarify the physical mechanism of the SERS behavior of the noble metallic PS, we used FDTD to simulate the local electric field characteristics of the substrate. A nano- 
resolution cubic grid in a three-dimensional system was established in the model, including the appropriate boundary conditions. The base model was placed in the air, the periodic boundary conditions close to the model were applied in the $\mathrm{x}$ and $\mathrm{y}$ directions, and the z-direction was surrounded by a perfectly matched layer (PML), in which a PML with a thickness greater than the wavelength was used to avoid false boundary reflection. The simulation of the regional background refractive index was set to 1, and the data of the Au and Ag material adopted the model data provided by the software itself. The excitation light was an ordinary incident plane wave, whose wavelength was $532 \mathrm{~nm}$, and it was oriented to illuminate the pyramid from the top (along the z-axis), where the polarization of the light was along the x-axis. A $2 \times 2$ pyramid array was selected as the basic framework. The PPS model was a positive pyramid array, as shown in Figure 3a. The thickness of the metal film was greater than its penetration depth, thus the part covered by the metal film was equivalent to a solid metallic pyramid for the simulation. The IPS model consisted of a noble metallic cuboid containing a hollow pyramid, the $x-z$ view of the model is shown in the Figure $3 b$. The pyramids in the PPS and IPS models have the same geometric parameters. The side length of each pyramid was $1 \mu \mathrm{m}$, and the slope angle $\theta$ of the pyramid was $54^{\circ}$, which was determined by the anisotropic corrosion of single-crystal silicon [45]. To avoid transmission due to the thinner thickness at pyramid joints, a $200 \mathrm{~nm}$ thick homogeneous metal layer was placed under the pyramid array. Figure 4 shows the two-dimensional electric field distribution diagram of the PPS and IPS along the $x-z$ plane.

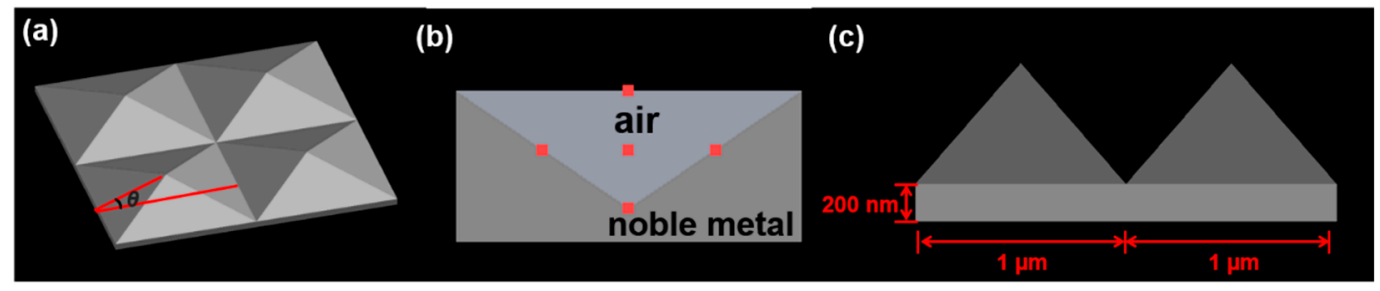

Figure 3. Schematic diagram of the noble metallic nano-pyramidal template. (a) PPS model; (b) IPS model in the $x-z$ plane; (c) side view of the pyramid array.

(a)

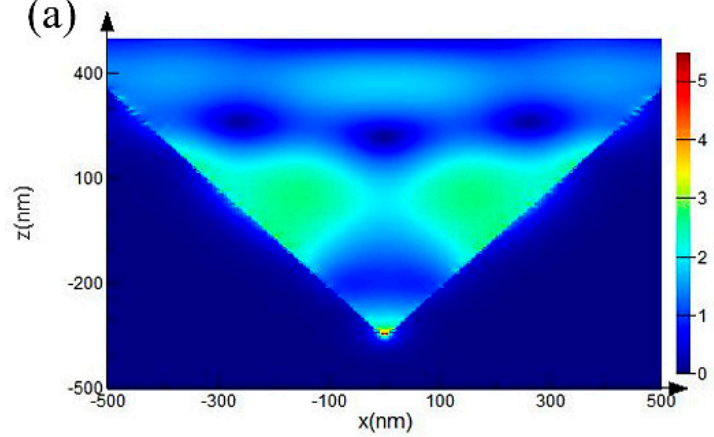

(c)

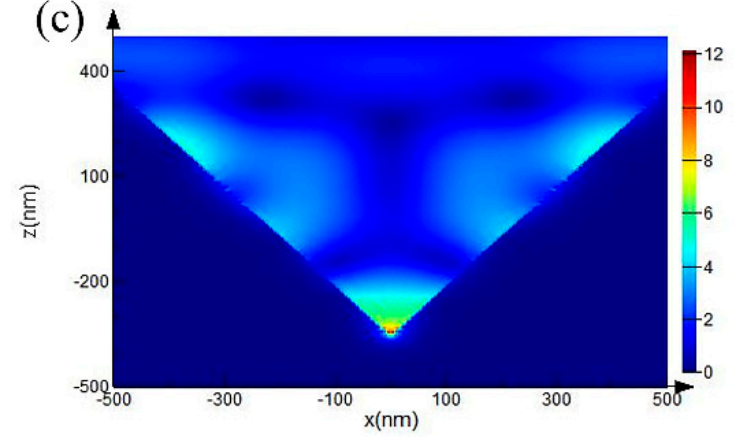

(b)

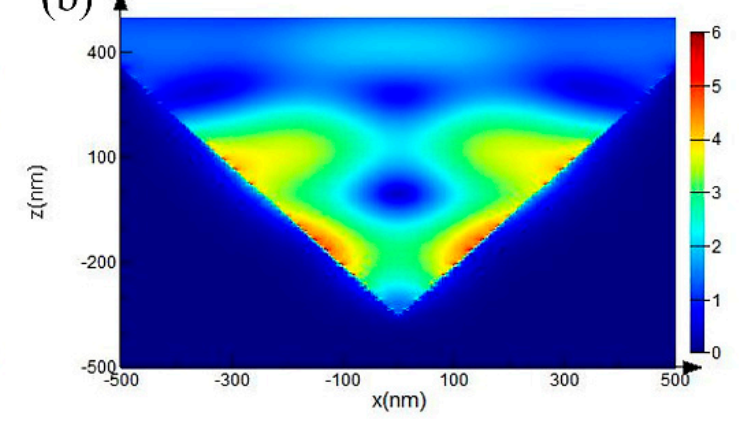

(d)

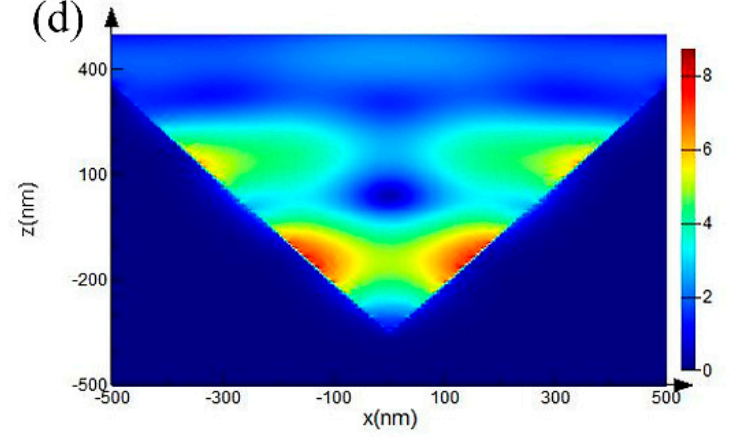

Figure 4. Electric field distribution diagram of a pyramid array in the x-z plane. (a) Au/PPS; (b) Au/IPS; (c) Ag/PPS; (d) Ag/IPS. 
Due to the polarization of the incident electric field, there will be two opposite charges on the two adjacent side walls, and they approach each other as they approach the bottom gap, forming a strong localized plasma [46,47]; the maximum strong electric field is at the bottom gap of the adjacent pyramids of PPS. The simulation results showed that the strengthening effect of Ag substrate is stronger than that of Au substrate, because the characteristic dipolar resonances for Ag nanoparticles are below $450 \mathrm{~nm}$, whereas for $\mathrm{Au}$ they are located around $550 \mathrm{~nm}$. Thus, a laser wavelength of $532 \mathrm{~nm}$ is near resonance for $\mathrm{Au}$, but well above resonance for Ag nanoparticles [48]. Therefore, Au has an absorption peak at $532 \mathrm{~nm}$, which leads to a poor SERS effect for Au substrate.

The influence of pits in the surface of IPS on the enhancement effect cannot be ignored, as shown in Figure $2 \mathrm{~g}, \mathrm{~h}$. The bonding force between Au and silicon is far less than that between Ag and silicon; therefore, during the stripping process, Au film can be completely stripped from the Si substrate, while the morphology is relatively flat. The opposite is true for Ag. Some Ag remains on the template surface, forming more uneven surfaces on $\mathrm{Ag} / \mathrm{IPS}$. This surface structure improves the enhancement effect and produces more "hot" sites for molecular detection than smooth conventional substrates.

Since these pits are too small to be measured, we applied some hemispheres to the model in Figure $4 \mathrm{~d}$. The radius of the hemispheres is different, which represents the randomness and contingency of the pits on a Ag film surface, as shown in Figure 5a. The radius of hemispheres 1-5 were $20 \mathrm{~nm}, 25 \mathrm{~nm}, 30 \mathrm{~nm}, 35 \mathrm{~nm}$, and $40 \mathrm{~nm}$, respectively, and the distances between adjacent hemispheres were equal. The radius of hemispheres 6-8 was $40 \mathrm{~nm}$, the distance between the adjacent hemispheres was equal and the depression depth was increasing. In line with the actual scale range, the larger the radius of the pit, the better the enhancement effect. Moreover, the deeper the pit, the better the enhancement effect; this is because the deeper pits form a sharper bulge with the side wall of the pyramid, resulting in stronger scattering. The probe molecule can easily reach these regions, Figure $5 \mathrm{~b}$ illustrates that the enhancement effect of Ag/IPS is better than that of Ag/PPS.

(a)

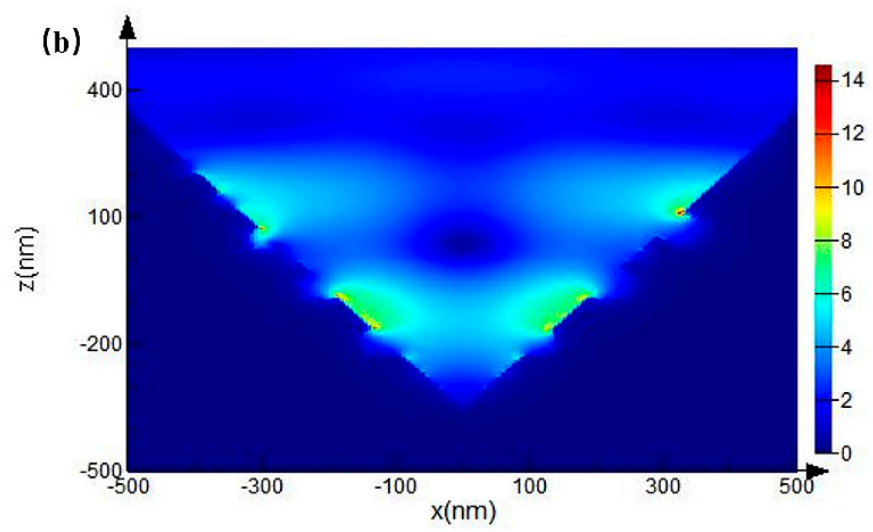

Figure 5. (a) Schematic diagram of a pit on the side wall of a pyramid; (b) Electric field distribution diagram of Ag/IPS with pits.

Moreover, the simple nano-particle dimer hotspot is only in the polarization direction of the incident light [49], while the IPS can change the polarization direction of the scattered light, as shown in Figure 6; under the same conditions, the electric field also distributes in the direction perpendicular to the polarization direction of the incident light. 


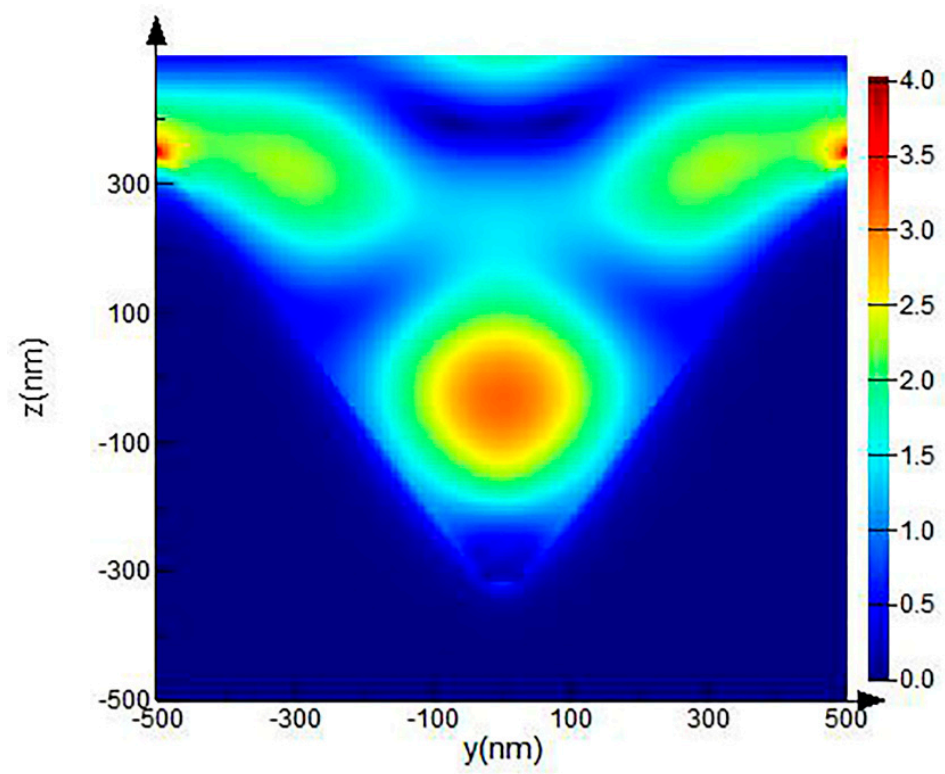

Figure 6. Electric field distribution diagram of Ag/IPS with pits in the y-z plane.

\subsection{Raman Spectra of R6G on Pyramidal Sensors}

In order to make the Raman spectra of probe molecules of all concentrations under the same laser power compare with the threshold range of the spectrum software, the laser defocusing mode with a laser spot of $2 \sim 3 \mu \mathrm{m}$ in diameter was used to make the laser focus on another plane outside the surface of the measured object, so as to reduce the excessive strong spectrum of the measured molecules with high concentration.

To study the SERS sensitivity of the improved structure, the spectra of four substrates were measured using R6G as a probe molecule, with the same concentration of $10^{-6} \mathrm{M}$, Figure 7 shows the SERS sensitivity measurement results obtained from four SERS substrates in R6G. The results show that all four substrates had a SERS effect. The enhancement effect of $\mathrm{Ag} / \mathrm{IPS}$ was far greater than that of Ag/PPS, which was consistent with the simulation results. It is worth noting that the enhancement effect of Au/PPS was better than that of $\mathrm{Ag} / \mathrm{PPS}$, which was contrary to the simulation results. This can be explained by the SPR effect. Previous studies showed that the SPR effect of Au occurs at $515.1 \mathrm{~nm}$ and that of Ag is at $395.8 \mathrm{~nm}$, so the SPR effect of Au is stronger than that of Ag [50]. Combined with the simulation, it can be inferred that the enhancement principles of PPS and IPS are different; the enhancement mechanism of IPS is dominated by scattering [51], while that of PPS is dominated by SPR [44].

In addition, there is no characteristic peak $\left(520 \mathrm{~cm}^{-1}\right)$ of $\mathrm{Si}$ at $520 \mathrm{~cm}^{-1}$ with PPS, which proves that the thickness of the noble metal film was greater than its penetration depth, so the enhancement performance of the substrate was not affected by the underlying Si template or UVcp.

To determine the lowest measurable concentration of R6G in the substrate, we collected the Raman signal of R6G solutions with different concentrations $\left(10^{-9} \mathrm{M} \sim 10^{-13} \mathrm{M}\right)$ under the best condition of Ag/IPS, as shown in Figure 8. The corresponding spectra of each concentration were the mean values of Raman peaks from four random locations. An error histogram plotted using the average of the four peaks at $611 \mathrm{~cm}^{-1}$ is shown in Figure 9 . In this experiment, the SERS signal of R6G solution was easily observed, and the Raman signal intensity increased with the increase of R6G concentration; the lowest detection concentration of the R6G probe on this substrate was as low as $10^{-12} \mathrm{M}$. Obviously, $\mathrm{Ag} / \mathrm{IPS}$ is very sensitive to SERS effects. The error of the characteristic peaks reflects the homogeneity of the SERS performance of the substrate structure. 


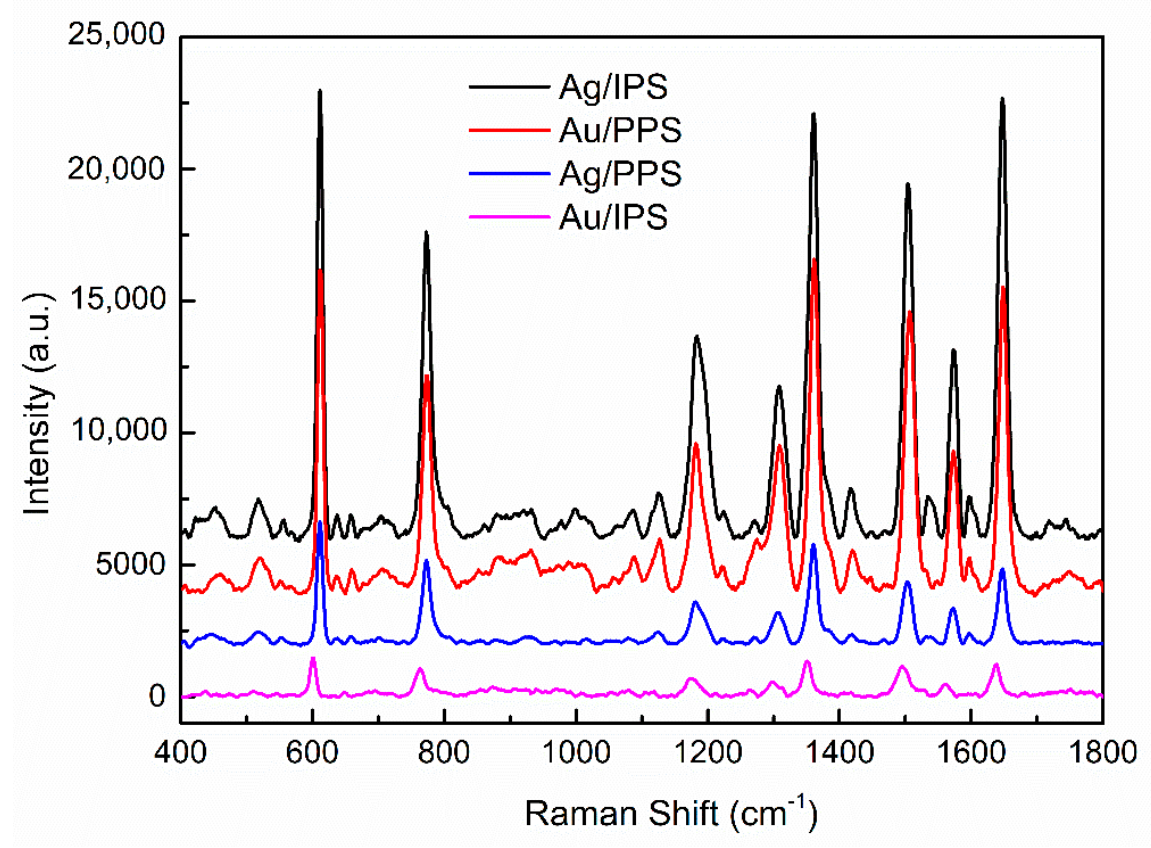

Figure 7. The SERS spectra of R6G $\left(10^{-6} \mathrm{M}\right)$ gathered on four pyramidal SERS substrates.

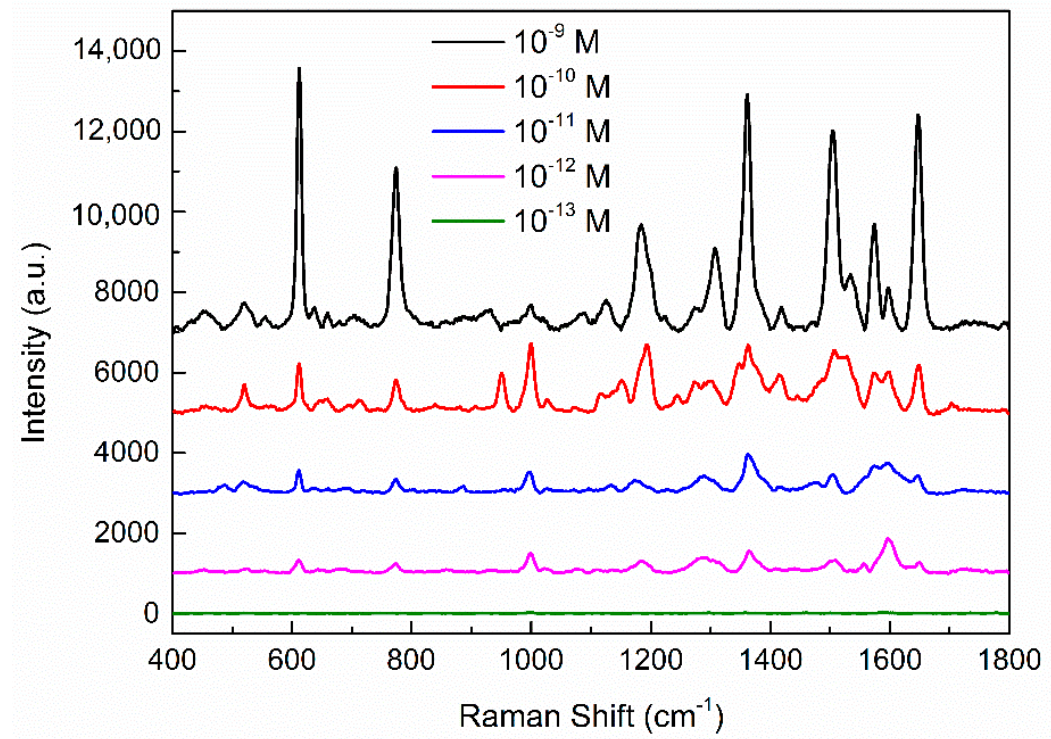

Figure 8. The SERS spectra of various concentrations of R6G solution (ranging from $10^{-9} \mathrm{M}$ to $10^{-13} \mathrm{M}$ ) gathered on Ag/IPS.

Finally, the stability of SERS substrate is very important. The SERS spectrum was measured again for the Ag/IPS soaked in R6G solution with a concentration of $10^{-6} \mathrm{M}$ and it was stored it in a 4-inch silicon wafer box and sealed at room temperature for three years. Figure 10 shows the SERS spectrum of the Ag/IPS, the red line is the Raman spectrum after preparation, while the black line is the Raman spectrum of the same substrate after three years. Three years later, the intensity of the Raman peak at $611 \mathrm{~cm}^{-1}$ was $29 \%$ of that of the initial result, which indicates that the proposed pyramidal SERS substrate has a good stability. 


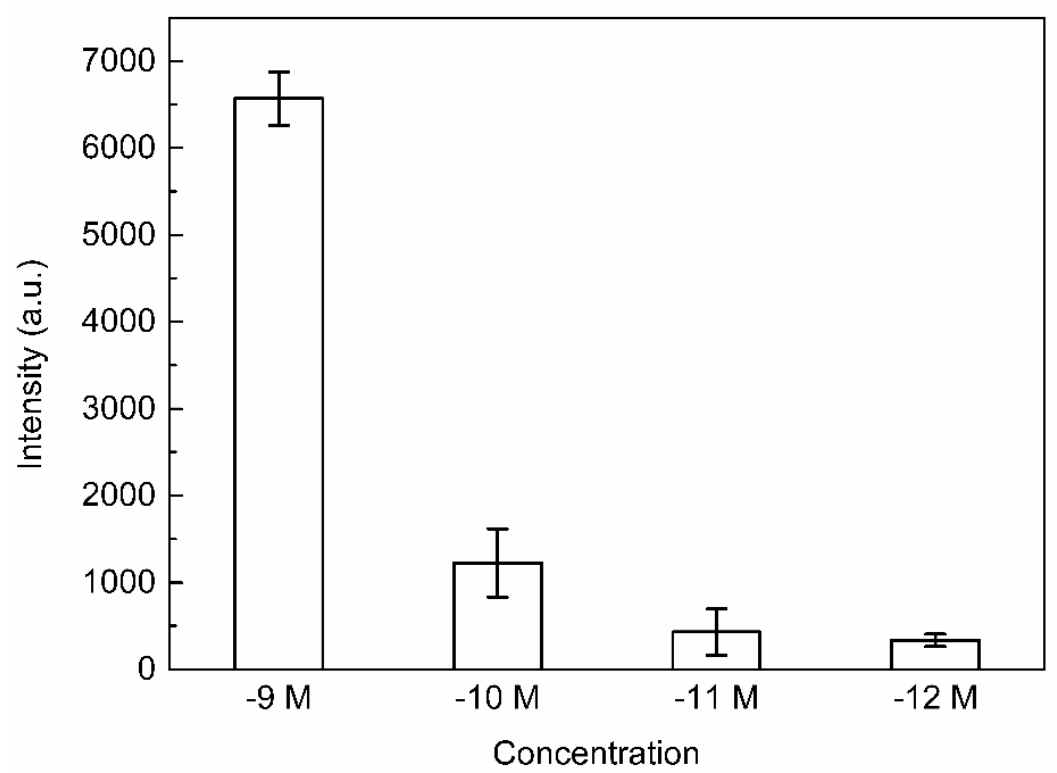

Figure 9. The histogram with errors of the average peak of R6G solution (ranging from $10^{-9} \mathrm{M}$ to $\left.10^{-12} \mathrm{M}\right)$ at $611 \mathrm{~cm}^{-1}$.

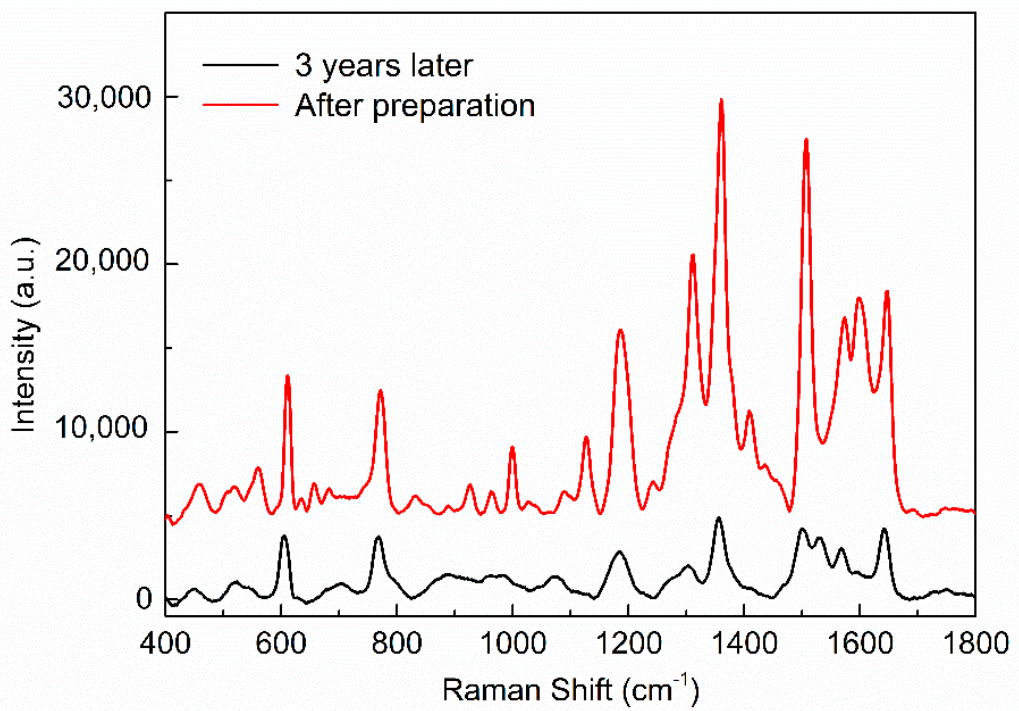

Figure 10. SERS spectra of Ag/IPS after preparation and 3 years later.

\subsection{SERS Using Pyramidal Sensors by Detection of Plasmid DNA}

The Ag/IPS was prepared by depositing an Ag layer with a thickness of $200 \mathrm{~nm}$ on the surface of the template and stripping it with UVcp. Plasmid DNA was used as a probe to detect the Raman signal on Ag/IPS. Due to the extremely short validity period of DNA, the immersion time of the substrate was shortened to 20 minutes. In order to exclude the influence of other extracts, the same enhanced substrate was immersed in LB broth under the same conditions, and its SERS signal was used as the control group; the SERS result is shown in Figure 11a. Furthermore, the intensities of the 681.0, 924.5, 1006.9, and $1412.8 \mathrm{~cm}^{-1}$ peaks in the four randomly selected spots are presented in Figure $11 \mathrm{~b}$, the average intensities were, respectively, $317.8,1044.4,850.3$, and 531.8 , with $13.12 \%, 6.59 \%$, $3.51 \%$, and $5.38 \%$ variation. Obviously, the intensity of Raman spectra was quite stable, with small fluctuations. Table 1 shows the band positions and their assignments to the spectra of plasmid DNA. Therefore, we can expect to detect plasmid DNA by using the pyramidal SERS substrate made using this method. Here are the meanings of the abbreviations: 
G: guanine; A: adenine; C: cytosine; T: thymine; Types of vibrations: $\delta$ = bending vibrations, $\delta_{s}=$ scissoring (in-plane bending) vibrations, $v=$ stretching vibration.

(a)

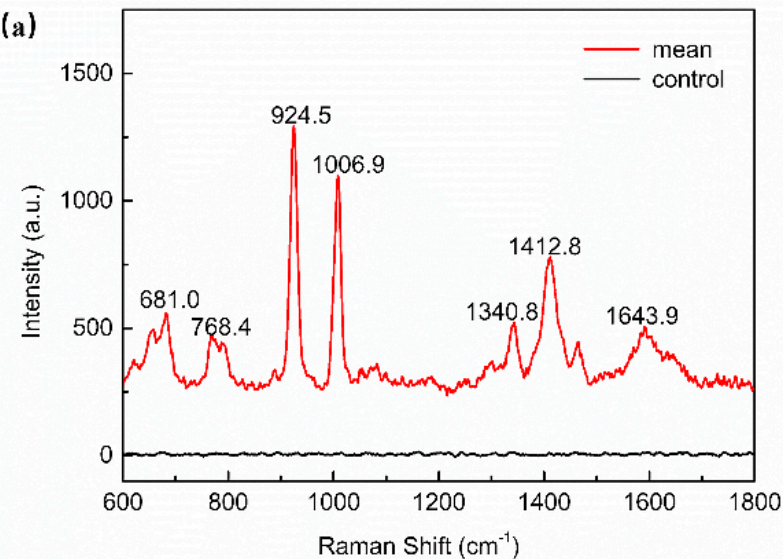

(b)

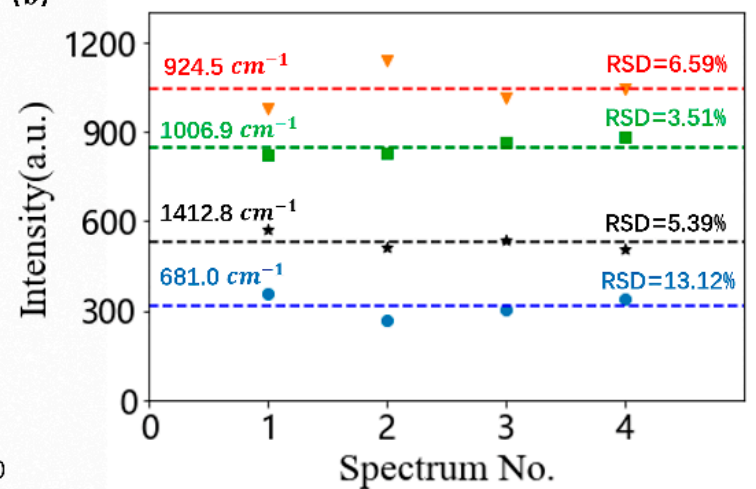

Figure 11. (a) The SERS spectra of plasmid DNA and the control group. (b) The intensity distributions of the 681.0, 924.5, 1006.9 , and $1412.8 \mathrm{~cm}^{-1}$ peaks of plasmid DNA in four randomly selected spots on the $\mathrm{Ag} / \mathrm{IPS}$, the dotted lines reveal the average intensities of each peak.

Table 1. Band positions and their assignments to spectra of plasmid DNA.

\begin{tabular}{cc}
\hline RamanShift $\left(\mathrm{cm}^{-1}\right)$ & Spectral Peak Assignment \\
\hline 681.0 & $682 \mathrm{~cm}^{-1}-\mathrm{C} 2^{\prime}$-endo/anti of G [52,53] \\
768.4 & $739 \sim 785 \mathrm{~cm}^{-1}$-Ring breathing of T [54] \\
924.5 & DNA phosphate skeletal motions [55] \\
1006.9 & $1005 \mathrm{~cm}^{-1}-5$-methylcytosine [56] \\
1340.8 & $1328 \mathrm{~cm}^{-1}-\mathrm{dT} \mathrm{in} \mathrm{C2'-endo/anti} \mathrm{of} \mathrm{S-type} \mathrm{[57]}$ \\
1412.8 & $1330 \mathrm{~cm}^{-1}-v(\mathrm{C}-\mathrm{N})$ of A, T [58] \\
1463.9 & $1412 \sim 1417 \mathrm{~cm}^{-1}-\delta(\mathrm{CH}), \delta(\mathrm{NH}), v(\mathrm{CN})[57]$ \\
$1463 \mathrm{~cm}^{-1}-\delta_{S}\left(\mathrm{~N}_{3}-\mathrm{H}\right), \delta\left(\mathrm{C}_{5}-\mathrm{CH}\right)$ of dT [59]
\end{tabular}

\section{Conclusions}

In summary, by combining the pyramidal template of silicon solar cell substrate, the flexible UVcp, and the noble metal film layer, we proposed a SERS substrate based on a template stripping method. R6G plays the role of the probe molecule, and the SERS results show that these substrates possess good sensitivity and specificity. Combined with the simulation of FDTD and the experimental results, we can explain the different enhancement mechanisms of noble metallic PPS and IPS. During experiments with different R6G concentrations, the Ag/IPS exhibited a good SERS behavior, which indicates that the noble metallic IPS is expected to be an effective substrate for unlabeled SERS detection. $\mathrm{Ag} / \mathrm{IPS}$ was also used to identify seven major molecular bands of low-concentration plasmid DNA. The low cost, good homogeneity, sensitivity, and stability verify that a noble metallic IPS is a promising candidate for label-free SERS detection in biological applications and mass screening in the future.

Author Contributions: Writing—original draft preparation, investigation, W.W.; conceptualization, writing-review and editing, validation, R.L.; software, J.L.; supervision, W.Z.; project administration, Z.J.; data curation, L.P.; supervision, M.C. All authors have read and agreed to the published version of the manuscript.

Funding: This work was financially supported by the Fundamental Research Funds for the Central Universities of China (Grant Nos. DUT19LK19 and DUT20YG132),National Natural Science Foundation of China (Nos. 51972039, 51803018), and LiaoNing Revitalization Talents Program (No. XLYC1902122). 
Conflicts of Interest: The authors declare no conflict of interest.

\section{References}

1. Wang, C.G.; Wu, X.Z. Orientation-dependent nanostructure arrays based on anisotropic silicon wet-etching for repeatable surface-enhanced Raman scattering. Nanoscale 2016, 8, 4672-4680. [CrossRef]

2. Liu, X.; Huang, M. Fabrication of a large-area, flexible micro-pyramid PET film SERS substrate and its application in the detection of dye in herbal tea. Laser Phys. Lett. 2018, 15, 55701. [CrossRef]

3. Oo, S.Z.; Chen, R.Y. Disposable plasmonic plastic SERS sensor. Opt. Express 2013, 21, 18484. [CrossRef]

4. Endo, T.; Yamada, H. Template stripping method-based Au nanoarray for surface-enhanced Raman scattering detection of antiepileptic drug. Micromachines 2020, 11, 936. [CrossRef] [PubMed]

5. Zhang, C.; Jiang, S.Z. SERS detection of R6G based on a novel graphene oxide/silver nanoparticles/silicon pyramid arrays structure. Opt. Express 2015, 23, 24811. [CrossRef]

6. Song, J.; Cheng, S. A novel surface-enhanced Raman scattering substrate: Diamond nanopit infilled with gold nanoparticle. Mater. Lett. 2014, 135, 214-217. [CrossRef]

7. Xia, M.; Zhang, P. Coupling SPP with LSPR for enhanced field confinement: A simulation study. J. Phys. Chem. C 2016, 120, 527-533. [CrossRef]

8. Cui, B.; Clime, L. Fabrication of large area nanoprism arrays and their application for surface enhanced Raman spectroscopy. Nanotechnology 2008, 19, 145302. [CrossRef]

9. Wang, Y.; Yang, Y. Rapidly fabricating large-scale plasmonic silver nanosphere arrays with sub-20 nm gap on Si-pyramids by inverted annealing for highly sensitive SERS detection. RSC Adv. 2017, 7, 11578-11584. [CrossRef]

10. Atanasov, P.A.; Nedyalkov, N.N. Ag and Au nanostructures for surface-enhanced Raman spectroscopy of Mospilan 20 SP (acetamiprid). J. Raman Spectrosc. 2020, 51, 2398-2407. [CrossRef]

11. Du, L.; Wang, Y. Preparation of Au nanocolloids by in situ dispersion and their applications in surface-enhanced Raman scattering (SERS) films. Ind. Eng. Chem. Res. 2016, 55, 6783-6791. [CrossRef]

12. Hering, K.; Cialla, D. SERS: A versatile tool in chemical and biochemical diagnostics. Anal. Bioanal. Chem. 2008, 390, 113-124. [CrossRef] [PubMed]

13. Kahl, M.; Voges, E. Periodically structured metallic substrates for SERS. Sens. Actuators B Chem. 1998, 51, 285-291. [CrossRef]

14. Ko, H.; Singamaneni, S. Nanostructured surfaces and assemblies as SERS media. Small 2008, 4, 1576-1599. [CrossRef]

15. Huang, J.; Zhao, Y. Ordered Ag/Si nanowires array: Wide-range surface-enhanced Raman spectroscopy for reproducible biomolecule detection. Nano Lett. 2013, 13, 5039-5045. [CrossRef]

16. Liu, A.; Zhang, S. Ag-coated nylon fabrics as flexible substrates for surface-enhanced Raman scattering swabbing applications. J. Mater. Res. 2020, 35, 1271-1278. [CrossRef]

17. Wang, Y.; Lu, N. Highly effective and reproducible surface-enhanced Raman scattering substrates based on Ag pyramidal arrays. Nano Res. 2013, 6, 159-166. [CrossRef]

18. Li, G.; Feng, W. Facile preparation of nanoporous Ag decorated with $\mathrm{CeO} 2$ nanoparticles for surface-enhanced Raman scattering. J. Mater. Res. 2019, 34, 2003-2013. [CrossRef]

19. Alba, M.; Pazos-Perez, N. Macroscale plasmonic substrates for highly sensitive surface-enhanced Raman scattering. Angew. Chem. Int. Ed. 2013, 52, 6459-6463. [CrossRef] [PubMed]

20. Lin, Y.; Liao, J. Focused ion beam-fabricated Au micro/nanostructures used as a surface enhanced Raman scattering-active substrate for trace detection of molecules and influenza virus. Nanotechnology 2011, 22, 185308. [CrossRef]

21. Yue, W.; Wang, Z. Electron-beam lithography of gold nanostructures for surface-enhanced Raman scattering. J. Micro-Mech. Micromech. 2012, 22, 125007. [CrossRef]

22. Abu Hatab, N.A.; Oran, J.M. Surface-enhanced Raman spectroscopy substrates created via electron beam lithography and nanotransfer printing. ACS Nano 2008, 2, 377-385. [CrossRef] [PubMed]

23. Okamoto, T.; Yamaguchi, I. Local plasmon sensor with gold colloid monolayers deposited upon glass substrates. Opt. Lett. 2000, 25, 372-374. [CrossRef]

24. Vieu, C.; Carcenac, F. Electron beam lithography: Resolution limits and applications. Appl. Surf. Sci. 2000, 164, 111-117. [CrossRef]

25. He, M.; Cao, B. Synthesis of multi-branched gold nanostructures and their surface-enhanced Raman scattering properties of 4-aminothiophenol. J. Mater. Res. 2019, 34, 2928-2934. [CrossRef]

26. Le Bris, A.; Maloum, F. Self-organized ordered silver nanoparticle arrays obtained by solid state dewetting. Appl. Phys. Lett. 2014, 105, 203102. [CrossRef]

27. $\mathrm{Wu}, \mathrm{W}$.; Hu, M. Cones fabricated by 3D nanoimprint lithography for highly sensitive surface enhanced Raman spectroscopy. Nanotechnology 2010, 21, 255502. [CrossRef]

28. Sun, C.; Linn, N.C. Templated fabrication of periodic metallic nanopyramid arrays. Chem. Mater. 2007, 19, 4551-4556. [CrossRef]

29. Melngailis, J.; Kamal, A.S.A. Focused ion beam lithography. Nucl. Instrum. Methods Phys. Res. 1993, 81, 1271-1280. [CrossRef]

30. Malek, K.; Brzózka, A. SERS imaging of silver coated nanostructured $\mathrm{Al}$ and $\mathrm{Al}_{2} \mathrm{O}_{3}$ substrates. The effect of nanostructure. J. Raman Spectrosc. 2014, 45, 281-291. [CrossRef]

31. Schmidl, G.; Dellith, J. Silicon template preparation for the fabrication of thin patterned gold films via template stripping. $J$. Micro-Mech. Micromech. 2015, 25, 125005. [CrossRef] 
32. Gong, J.; Lipomi, D.J. Micro- and nanopatterning of inorganic and polymeric substrates by indentation lithography. Nano Lett. 2010, 10, 2702-2708. [CrossRef] [PubMed]

33. Wang, P.; Liang, O. Ultra-sensitive graphene-plasmonic hybrid platform for label-free detection. Adv. Mater. 2013, 25, 4918-4924. [CrossRef] [PubMed]

34. Xie, W.; He, S. Fabrication of a trans-scale bimetallic synergistic enhanced Raman scattering substrate with high surface-en hanced Raman scattering activity. Anal. Methods 2015, 7, 1676-1679. [CrossRef]

35. Li, J.F.; Huang, Y.F. Shell-isolated nanoparticle-enhanced Raman spectroscopy. Nature 2010, 464, 392-395. [CrossRef] [PubMed]

36. Krohne-Nielsen, P.; Novikov, S.M. Surface-enhanced Raman microscopy of hemispherical shells stripped from templates of anodized aluminum. J. Raman Spectrosc. 2012, 43, 834-841. [CrossRef]

37. Klemme, D.J.; Johnson, T.W. Self-aligned grating couplers on template-stripped metal pyramids via nanostencil lithography. Appl. Phys. Lett. 2016, 108, 213106. [CrossRef] [PubMed]

38. Martina, M.; Fleischer, M. Template stripping and bonding of smooth probes with nanoscale features for tip-enhanced Raman spectroscopy. Microelectron. Eng. 2017, 171, 31-36. [CrossRef]

39. Tiefenauer, R.F.; Tybrandt, K. Fast and versatile multiscale patterning by combining template-stripping with nanotransfer printing. ACS Nano 2018, 12, 2514-2520. [CrossRef]

40. Rubio-Lara, J.A.; Bergler, F. Ultraflat gold QCM electrodes fabricated with pressure-forming template stripping for protein studies at the nanoscale. Langmuir 2019, 35, 8889-8895. [CrossRef]

41. Zhang, J.; Winget, S.A. Ag@Au concave cuboctahedra: A unique probe for monitoring Au-catalyzed reduction and oxidation reactions by surface-enhanced Raman spectroscopy. ACS Nano 2016, 10, 2607-2616. [CrossRef]

42. Pashaee, F.; Tabatabaei, M. Tip-enhanced Raman spectroscopy: Plasmid-free vs. plasmid-embedded DNA. Analyst 2016, 141, 3251-3258. [CrossRef]

43. Mirajkar, S.; Dhayagude, A. Distinguishing genomic DNA of Brassica juncea and Arabidopsis thaliana using surface-en hanced Raman scattering. J. Raman Spectrosc. 2019, 51, 89-103. [CrossRef]

44. Li, R.; Lei, J. Hybrid 3D SERS substrate for Raman spectroscopy. Chem. Phys. Lett. 2020, 754, 137733. [CrossRef]

45. Ferchichi, A.; Laariedh, F. Fabrication of disposable flexible SERS substrates by nanoimprint. Microelectron. Eng. 2015, 140, 52-55. [CrossRef]

46. Li, R.; Li, H. Surface-enhanced Raman scattering from rhodamine 6G on gold-coated self-organized silicon nanopyramidal array. J. Mater. Res. 2013, 28, 3401-3407. [CrossRef]

47. Vernon, K.C.; Davis, T.J. Physical mechanisms behind the SERS enhancement of pyramidal pit substrates. J. Raman Spectrosc. 2010, 41, 1106-1111. [CrossRef]

48. Liberman, V.; Sworin, M. Nonlinear bleaching, absorption, and scattering of 532-nm-irradiated plasmonic nanoparticles. J. Appl. Phys. 2013, 113, 53107. [CrossRef]

49. Liu, S.; Wang, X. The fabrication of a high-sensitivity surface-enhanced Raman spectra substrate using texturization and electroplating technology. Appl. Surf. Sci. 2019, 490, 109-116. [CrossRef]

50. Girão, A.V.; Pinheiro, P.C. Tailoring gold and silver colloidal bimetallic nanoalloys towards SERS detection of rhodamine 6G. RSC Adv. 2017, 7, 15944-15951. [CrossRef]

51. Lee, Y.; Kamal, A.S.A. Gap plasmons multiple mirroring from spheres in pyramids for surface-enhanced Raman scattering. ACS Photonics 2016, 3, 2405-2412. [CrossRef]

52. Lipiec, E.; Sekine, R. Molecular Characterization of DNA double strand breaks with tip-enhanced Raman scattering. Angezw. Chem. Int. Ed. 2014, 53, 169-172. [CrossRef] [PubMed]

53. Olsztyńska-Janus, S.; Gasior-Głogowska, M. Spectroscopic techniques in the study of human tissues and their components. Part II: Raman spectroscopy. Acta Bioeng. Biomech. 2012, 14, 121-133.

54. Gupta, S.; Banaszak, A. Detection of DNA bases and environmentally relevant biomolecules and monitoring ssDNA hybridization by noble metal nanoparticles decorated graphene nanosheets as ultrasensitive G-SERS platforms. J. Raman Spectrosc. 2021, 52, 930-948. [CrossRef]

55. Benedetti, E.; Bramanti, E. Determination of the relative amount of nucleic acids and proteins in leukemic and normal lymphocytes by means of fourier transform infrared microspectroscopy. Appl. Spectrosc. 1997, 51, 792-797. [CrossRef]

56. Moisoiu, V.; Stefancu, A. SERS assessment of the cancer-specific methylation pattern of genomic DNA: Towards the detection of acute myeloid leukemia in patients undergoing hematopoietic stem cell transplantation. Anal. Bioanal. Chem. 2019, 411, 7907-7913. [CrossRef]

57. Andrushchenko, V.; Tsankov, D. Spectroscopic detection of DNA quadruplexes by vibrational circular dichroism. J. Am. Chem. Soc. 2011, 133, 15055-15064. [CrossRef] [PubMed]

58. Dovbeshko, G.I.; Gridina, N.Y. FTIR spectroscopy studies of nucleic acid damage. Talanta 2000, 53, 233-246. [CrossRef]

59. Ponkumar, S.; Duraisamy, P. Structural analysis of DNA interactions with magnesium ion studied by Raman spectroscopy. Am. J. Biochem. Biotechnol. 2011, 7, 135-140. 